\title{
Building of laser-assisted micromachining temperature field
}

\author{
Jinkai Xu, Chuanpeng Chu, Zhilong Zhao, Xingxing Wang \\ Changchun University of Science and Technology \\ Changchun, China \\ xujinkai2000@163.com
}

\begin{abstract}
Aims: Laser-assisted micromachining is a promising technique to improve the machinability of various difficult-to-machine materials, building temperature field mathematical models of laser-assisted micromachining system can significantly reduce human and material resources in actual processing and improve efficiency. Method: This paper based on ANSYS parametric design language described the temperature field mathematical models' building process in laser-assisted micromachining system in details. Adopted Gaussian distribution model as the laser source of heat flow load, analyzed the calculation methods of heat flux values corresponding to different laser power, stated the construction method of laser light spot in mathematical models and the loading principle of moving light source in simulation. Result: By building mathematical models of laser-assisted micromachining system, successfully obtained the temperature contours and the temperature contours map.
\end{abstract}

Key words-laser-assisted micromachining; APDL; mathematical models; temperature field

\section{INTRODUCTION}

The development of aerospace, defense and microelectronics industry, puts forward a variety of new and special requirements to the performance of product parts, at the same time the demand for high precision 3D miniaturized components are increasingly urgent. To meet the mechanical parts' strength, stiffness and life criterion requirements in special circumstance, multitudes of engineering materials with high strength, high hardness, corrosion resistance, and possess many other excellent performance are more widely applied to the field of parts preparation. To these high-strength materials conventional processing technology has exposed serious drawbacks, such as severe tool abrasion, low processing efficiency, surface quality defects and so on. While utilizing an external heat source raising the cutting zone temperature to soften the cutting materials which is called thermally assisted processing technology [1], is an effective solution to these tough drawbacks. In thermally assisted machining, due to laser possesses strong monochromaticity, directivity, coherency, and high brightness as well as wide power regulation range features, made laser became an ideal heat source selection. Laser as the heat source own the following characteristics: transmission easy, acting site can be arbitrarily selected, light spot shape and size can be adjusted, and can control the depth and shape of acted area; high energy density, easy to control the heating time, able to locally heating, only heating the necessary parts and heating up fast.

Support by National Nature Science Foundation of China (NSFC, grant No. 51275056 and grant No. 51305043).
Laser Assisted Micromachining (LAMM) is focused laser beam on the workpiece surface prior to the cutting tool, before the material is removed immediately heating it locally to higher temperature, soften the materials, to reduce the cutting force, improve processing efficiency, surface quality and lower costs. LAMM schematic drawing shows in figure 1. And experiment testified LAMM can effectively reduce material strength, decrease cutting force, enhance material ductility, made processed surface more smooth, which can be seen in figure 2 , and made chips from break chips into continuous chips, which can be seen in figure 3 .

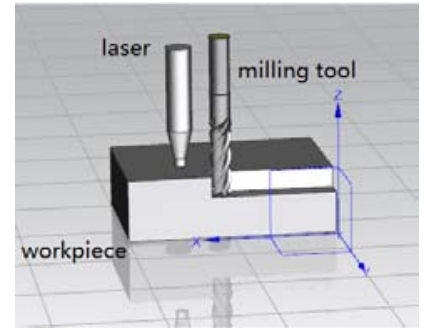

Fig. 1. Schematic drawing of LAMM.

Temperature field is the main physical characteristic appeared after laser heating materials [3], temperature control is the key of LAMM [4], it directly determines whether high precision, good surface quality products can be produced or not Overheating will cause material burnt, impact the processed workpieces surface quality, and underheating will greatly reduce the effect of laser-assisted, so it is critical to determine how the temperature changes and distributes, and get accurate temperature parameters after the material heated by laser. LAMM is a quickly heating local to high temperature and then naturally cooling down process, with the movement of heat source the workpiece entire temperature will changes drasticly along with time and space, it is a typical nonlinear transient heat transfer process [6]. Temperature field of LAMM is a collection of each moment the object in every point temperature parameters, in general temperature field is a function of coordinates and time [7], which can be expressed by the following equation:

$$
\mathrm{T}=\mathrm{f}(\mathrm{x}, \mathrm{y}, \mathrm{z}, \mathrm{t})
$$

In the process of LAMM, the workpiece temperature distribution must be measured and controlled effectively [8], so that to select the best laser parameters and achieve the desired effect of LAMM. As the laser heating speed fast and action range small, using experimental method to measure the temperature distribution will consume vast human and material 
resources, at the same time because of the response rate with a time delay, adopt thermocouple to accurately measure the

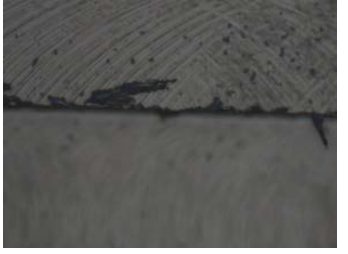

(a) without laser

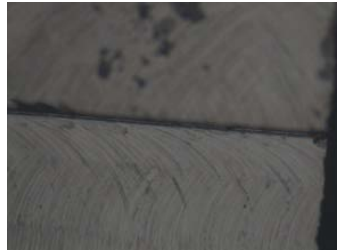

(b) added laser
Fig.2.

\section{THERMAL TRANSFER MODE}

Temperature difference is the driving force in thermal transfer process, thermal transfer always from high temperature to low temperature spontaneously [7], in the process of laser heating, the workpiece temperature of heated site increase transiently, there is heat transfer internal the workpiece, between the workpiece surface and the surroundings exists thermal convection and thermal radiation transient heat transfer process, shows in figure 4.

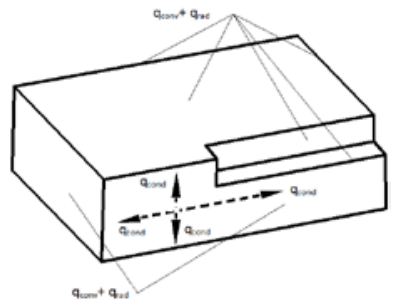

Fig. 4. Schematic drawing of thermal transfer mode.

Heat conduction [7] is between the parts of object does not occur relative displacement, heat transfer depend on the thermal motion of molecules, atoms, free electrons and other microscopic particles, differential equation of heat conduction as follows:

$$
\rho c \frac{\partial \mathrm{t}}{\partial \tau}=\frac{\partial}{\partial \mathrm{x}}\left(\lambda \frac{\partial \mathrm{t}}{\partial \mathrm{x}}\right)+\frac{\partial}{\partial \mathrm{y}}\left(\lambda \frac{\partial \mathrm{t}}{\partial \mathrm{y}}\right)+\frac{\partial}{\partial \mathrm{z}}\left(\lambda \frac{\partial \mathrm{t}}{\partial \mathrm{z}}\right)+\dot{\Phi}
$$

$\rho$ represent density, c represent specific heat capacity, $\Phi$ represent per unit time and per unit volume generated heat by inner heat source, $\tau$ represent time, $\lambda$ represent coefficient of thermal conductivity, $\frac{\partial \mathrm{t}}{\partial \mathrm{x}}$ represent temperature change rate in $\mathrm{x}$ direction, $\frac{\partial t}{\partial y}$ represent temperature change rate in $\mathrm{y}$ direction, $\frac{\partial \mathrm{t}}{\partial \mathrm{z}}$ represent temperature change rate in $\mathrm{z}$ direction.

Heat convection [7] is a heat transfer process because of the relative displacement between various parts of the fluid caused by fluid macroscopic motion, lead to cold and hot fluid blended with each other, heat convection generally applied as a surface boundary conditions. Heat convection in Newton's cooling equation can be described as:

$$
\mathrm{q}=\mathrm{h}_{\mathrm{f}}\left(\mathrm{T}_{\mathrm{s}}-\mathrm{T}_{\mathrm{B}}\right)
$$

$\mathrm{q}$ represent heat convection values, $h_{\mathrm{f}}$ represent convection coefficient of thermal conductivity, $T_{S}$ represent surface temperature of workpiece, $T_{B}$ represent temperature of surrounding fluid. transient temperature field is not easy [9]. However, numerical simulation is a simple and effective way.

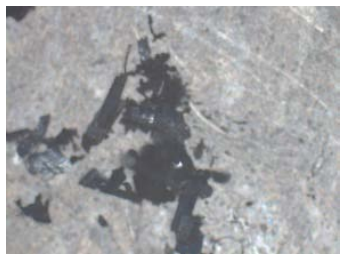

(a) without laser

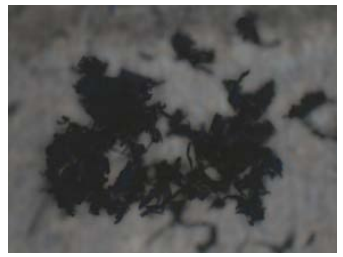

（b） added laser
Fig. 3.

Energy transferred by electromagnetic waves is called radiation, thermal radiation [10] is the radiation phenomenon caused by hot. Thermal radiation are different from heat conduction and heat convection, each object will emit radiation, at the same time it also absorb radiation, and the process of thermal radiation does not rely on any medium. Workpiece's net heat transmission to the surrounding open space after laser heating, can be calculated by the following Stephen-Boltzmann equation:

$$
\mathrm{Q}=\varepsilon \sigma \mathrm{A}\left(\mathrm{T}_{1}{ }^{4}-\mathrm{T}_{2}{ }^{4}\right)
$$

$\varepsilon$ represent emissivity, $\sigma$ represent Stephen-Boltzmann constant, it is about $5.67 \times 10^{-8} \mathrm{~W} / \mathrm{m}^{2} \cdot \mathrm{K}^{4}$, A represent the area of radiation surface, $T_{1}$ represent thermodynamic temperature of radiation surface, $T_{2}$ represent thermodynamic temperature of space.

\section{TEMPERATURE FIELD BUILDING PROCESS AND LASER PARAMETERS SETTING}

Based on ANSYS Parametric Design Language (APDL), adopted Finite Element Method (FEM) in numerical modeling built the LAMM mathematical model of temperature field, and the flow path shows in figure 5.

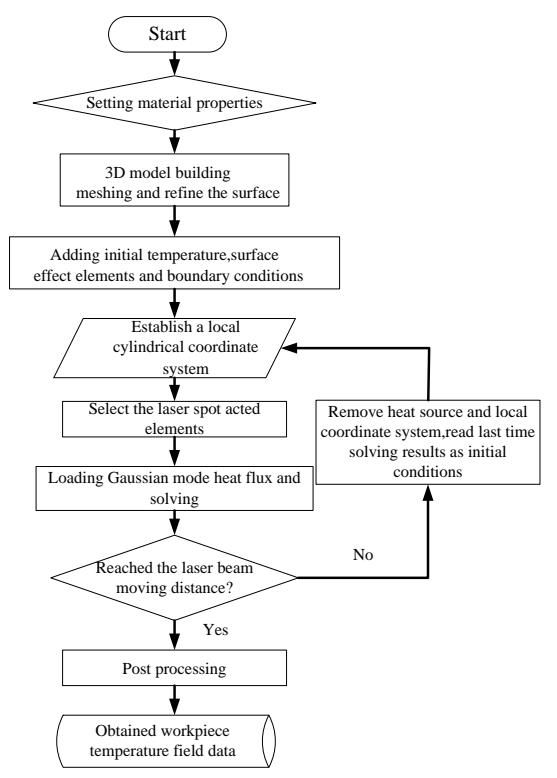

Fig. 5. Flow path of temperature field building.

Before completed the workpiece 3D model construction, material property, element type and element real constant must be set. Adopt solid element "Solid70" and surface effect 
element "Surf152" to apply heat flux load and heat convection, thermal radiation boundary conditions [11]. As laser heating time short, and the surface temperature of laser irradiation region increasing rapidly, so the surface layer temperature gradient is large, it require the meshing of the workpiece's laser heated surface to be denser. For the purpose of reducing the amount of calculation, at the same time ensure the results calculation accuracy, after meshing the workpiece surface which laser acted should be refined.

In the process of laser heating, laser beam energy as an external flux vector continuously apply to the light spot irradiation region, light spot position is a continuous function of time and coordinates. According to the finite element discrete thought, a round face of certain radius can be divided into a collection of several polygon, the round light spot of laser also can be divided into a plurality of discrete finite elements included within the scope of the spot diameter. Through establish a cylindrical coordinate system in the spot center coordinate position, in this coordinate system select the elements included in the light spot radius, the outline composed by the selected elements is the laser spot profile, shows in figure 6 , then on these selected elements apply the heat flux

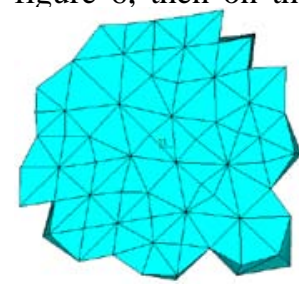

(a) Profile of light spot

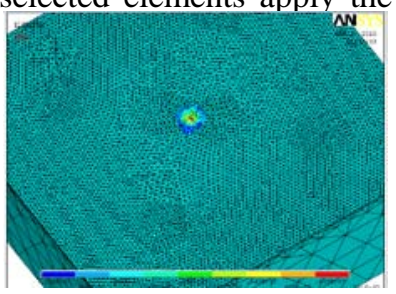

(b) Profile of light spot in workpiece
Fig. 6.

corresponding to the laser power, this process is equivalent to the heating process in the laser beam active area. By setting different radius values to achieve change the light spot size. The laser light source in actual process is continuously moving with the cutting process carrying out, in numerical simulation the laser source's continuously moving process can be accomplished by changing the cylindrical coordinate system position in Cartesian coordinate system continuously, that is utilizing small step intermittent moving heat source to approximately simulate the laser's continuous movement during the workpiece's machining process [12]. If in the cutting process the light source of laser moving along the direction of $\mathrm{x}$, the cutting length is $\mathrm{s}$, and the moving speed of light source is $\mathrm{v}$, then in numerical simulation adopt $\Delta \mathrm{x}$ as the value of step distance, in each step the residence time is $t$, $\mathrm{t}=\Delta \mathrm{x} / \mathrm{v}$, to approximately simulate the continuous motion of light source, the motion process shoes in figure 7.The motion

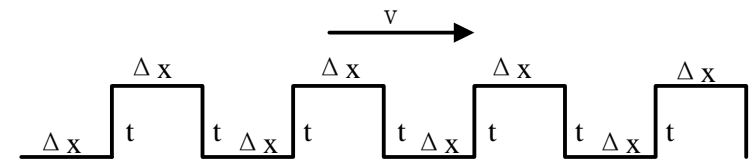

Fig. 7. Laser source motion process.

number of light source $n=s / \Delta x$, by shorten $\Delta x$, enlarge $n$ can make the simulation more close to the continuous movement of laser.
The heat flux of laser spot on the workpiece surface is fundamental mode Gaussian distribution, the value of heat flux $\mathrm{q}$ with the increasing distance from the center of the spot decrease with the relationship of index, and the value of heat flux q can be expressed as:

$$
\mathrm{q}=\frac{2 \alpha \mathrm{P}}{\pi \mathrm{R}^{2}} \exp \left(\frac{-2 \mathrm{r}^{2}}{\mathrm{R}^{2}}\right)
$$

$\alpha$ represent workpiece absorption rate to laser, $\mathrm{P}$ represent laser power, $\mathrm{R}$ represent the radius of laser spot, $\mathrm{r}$ represent the distance from the spot center. Figure 8 shows the variation trend of the heat flux value in the laser spot area. By loading the heat flux value corresponding to different laser power on workpiece surface, the corresponding workpiece temperature distribution parameters can be obtained.

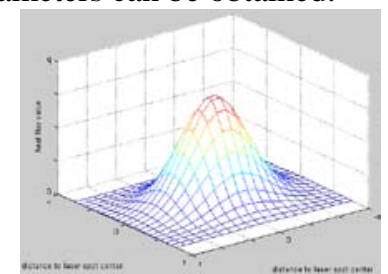

Fig. 8. Laser spot heat flux variation trend.

After accomplished the setting of material property, the selection of element type, the workpiece 3D model building and meshing, through apply initial temperature and boundary conditions, setting the laser power, laser spot radius, laser spot motion speed and other solving conditions, then by solving to the model and post-processing, the corresponding workpiece temperature field to laser parameters can be obtained. Figure 9 shows the temperature contours and the temperature contours map obtained by numerical simulation to $45 \#$ steel under certain laser parameters, the temperature distribution results indicates, after the moving laser beam action the temperature contours map of the workpiece is elongated ellipse, temperature away from the laser beam action region changes relatively slow, temperature gradient is small, temperature near the laser beam action region changes fast, temperature gradient relatively large.

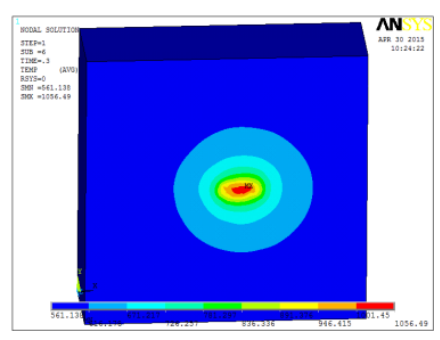

(a) temperature contours

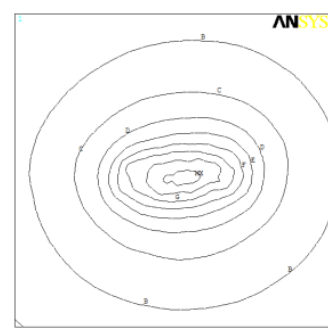

(b) temperature contours map Fig. 9.

\section{CONCLUSION}

LAMM technology in processing the engineer materials with high strength, high hardness, corrosion resistance, etc properties is an effective way, to those difficult processing 
materials LAMM technology is a critical method to improve the low efficiency and high cost drawbacks. In actual process laser power, laser spot radius and laser light source motion speed to the workpiece temperature distribution is mutual restraint, mutual influence. Rely on ANSYS software to simulate the temperature field of laser heated workpiece, can obtain the temperature distribution parameters corresponding to different laser parameters, provided a basis for the reasonable selection of laser spot radius, laser light source motion speed and laser power in actual processing, it can effectively reduce the actual processing workload, improve processing efficiency.

\section{REFERENCES}

[1] Ye Bangyan. Heating Cut and Development. Machinery Development, 1995, pp. 10-12.

[2] König W,Treppe F,Zaboklicki K.Laserunterstütztes- -fräsen.VDI-Z, vol. 134, pp. 43-48, 1992.

[3] Zhang Yingcong, Sheng Hua , Zhu Rihong. Three-Dimensional Temperature Field of Material Irradiated by Continuous Wave Laser. Chinese Journal of Lasers, vol. 40, pp. 08060021-08060025, 2013.
[4] Ao Mingwu, Zhang Hongquan, Li Bo. Laser Heat Assisted Machining and Its Developing. Modern Machinery, 2003, pp. 81-82.

[5] Zhao Fengyan. Study of Thermal Effect on Metal and Semiconductor Materials Irradiated by Continuous Wave Laser. Changchun : Changchun University of Science and Technology, 2011.

[6] Chen Pei, Jin Xiangzhong. Temperature Field Numerical Simulation of Laser-assisted Machining. Machinery Manufacturing, vol. 43, pp. 3234, 2005.

[7] Yang Shiming, Tao Wenquan. Heat Transfer. Beijing : Higher Education Press, 2006

[8] Shi Yuhao, Liu Changyi, Shen Xiaodong. Finite Element Analysis of Laser-assisted Milling Titanium TC4. Machinery Manufacturing, vol. 52, pp. 60-63, 2014.

[9] Zhu Zuchang, Wang Weirong, Yu Shaoluo. The Austenized Transformation in Laser Heating Processing. Chinese Journal of Lasers, vol. A21, pp. 846-851, 1994.

[10] Ge Shaoyan , Na Hongyue. Thermal Radiation Properties and Measurement. Beijing: Science Press, 1989.

[11] Hu Renxi, Kang Shiting. ANSYS14.0 Thermodynamic Finite Element Analysis From Entry to Master. Beijing: Machinery Industry Press, 2013.

[12] Guo Qing, Jiang Wanqing. Numerical Simulation of Laser Welding Temperature Field. Aviation Maintenance \& Engineering, 2004, pp. 2829. 